\title{
ENFERMEIROS NO FORTALECIMENTO DA REDE DE SAÚDE NUMA CIDADE DO TRIÂNGULO SUL/MINAS GERAIS
}

\section{NURSES IN THE STRENGTHENING OF THE HEALTH NETWORK IN A CITY IN THE SOUTH TRIANGLE/MINAS GERAIS}

\section{ENFERMEROS EN EL FORTALECIMIENTO DE LA RED DE SALUD EN UNA CIUDAD DE TRIANGULO SUL/MINAS GERAIS}

Marciana Fernandes Moll ${ }^{1}$, Mariana Bonomi Goulart ${ }^{2}$, Aloma Pegorini Caprio ${ }^{2}$, Carla Aparecida Arena Ventura ${ }^{3}$

\section{RESUMO}

Objetivo: investigar as ações desenvolvidas por Enfermeiros para fortalecer da Rede de Atenção à Saúde em uma cidade do Triângulo Sul de Minas Gerais. Método: estudo descritivo, exploratório, de abordagem qualitativa, realizado com 12 enfermeiros das Estratégias de Saúde da Família de uma cidade do Triângulo Mineiro. Os dados foram obtidos por meio de entrevista individual semiestruturada e foram submetidos à Análise Temática. Resultados: as temáticas identificadas foram: descontinuidade da comunicação entre os níveis de atenção à saúde; parceria com alguns serviços especializados; promoção da saúde da população; atendimento às gestantes. Conclusão: dentre as ações desenvolvidas para o fortalecimento da Rede, destacam-se: promoção e proteção da saúde, ações educativas sobre o fluxo e a operacionalização da Rede, e parcerias com alguns serviços especializados e setor social. Contudo, ainda se faz necessário o estabelecimento de parcerias com outros serviços de saúde que trabalhem com a saúde do trabalhador, saúde do idoso, saúde da criança e outros setores do desenvolvimento e educação.

Descritores: Enfermagem; Rede de cuidados continuados de saúde; Sistema único de saúde.

\section{ABSTRACT}

Objective: To investigate the actions developed by Nurses to strengthen the Health Care Network in a city in the South Triangle of Minas Gerais. Method: a descriptive, exploratory, qualitative study carried out with 12 nurses from the Family Health Strategies of a city of the Triângulo Mineiro. The data were obtained through a semi-structured individual interview and were submitted to the Thematic Analysis. Results: the themes identified were: discontinuity of communication between levels of health care; partnership with some specialized services; health promotion of the population; care for pregnant women. Conclusion: Among the actions developed to strengthen the Network, the following stand out: promotion and protection of health, educational actions on the flow and operation of the Network, and partnerships with some specialized services and social sector. However, it is still necessary to establish partnerships with other health services that work with worker health, elderly health, child health and other development and education sectors.

Keywords: Nursing; Network of continued health care; Health Unic System.

\section{RESUMEN}

Objetivo: investigar las acciones desarrolladas por enfermeros para el fortalecimiento de la Red de Atención a Salud en una ciudad de Triangulo Sul de Minas Gerais. Método: estudio descriptivo, exploratorio, de abordaje cualitativo, realizado con 12 enfermeros de Estrategias de Salud Familiar de una ciudad de Triángulo Minero. Los datos fueron recogidos por medio de entrevista individual semiestructurada y fueron sometidos a Análisis Temático. Resultados: fueron identificados los temas: discontinuidad de la comunicación entre los níveles de atención a la salud; asociación con algunos servicios especializados; promoción de la salud de la población; cuidado a mujeres embarazadas. Conclusión: Entre las acciones desarrolladas para el fortalecimiento de la Red, son destacadas: promoción y protección de la salud; acciones educativas sobre el flujo y la operacionalización de la Red y asociación con algunos servicios especializados y sector social. Sin embargo, aún es necesario el establecimiento de asociaciones con otros servicios de salud que trabajen con la salud del trabajador, salud del anciano, salud de los niños y otros sectores de desarrollo y educación.

Descriptores: Enfermería; Red de cuidados continuados de salud; Sistema único de salud.

${ }^{1}$ Graduada em Enfermagem. Doutora em Enfermagem Psiquiátrica pela Universidade de São Paulo. Docente na Universidade de Uberaba. ${ }^{2}$ Graduanda em Enfermagem pela Universidade de Uberaba. ${ }^{3}$ Graduada em Direito. Doutora em Administração pela Universidade de São Paulo. Docente na Universidade de São Paulo.

Como citar este artigo:

Moll MF, Goulart MB, Caprio AP, et al. Enfermeiros no Fortalecimento da Rede de Saúde em uma Cidade do Triângulo Sul/ Minas Gerais. 2018;8:e2393. Revista de Enfermagem do Centro-Oeste Mineiro. [Access ]; Available in: DOI: https://doi.org/10.19175/recom.v7i0.2393 


\section{INTRODUÇÃO}

As Redes de Atenção à Saúde (RAS) compreendem serviços e ações que intervém nos processos de saúde-doença com o auxílio de recursos tecnológicos, logísticos e de gestão, para assegurar a integralidade do cuidado e melhorar o acesso, a equidade e a eficácia propostos pelo Sistema Único de Saúde (SUS) ${ }^{(1)}$. Nesse sentido, a RAS é constituída por três elementos: população, estrutura operacional e modelo de atenção à saúde ${ }^{(2)}$.

Contudo, a articulação entre esses elementos ainda está fragilizada e, por isso são propostas ações prioritárias para a consolidação da RAS, das quais se destacam: comunicação entre os serviços de saúde existentes nos diferentes níveis de atenção à saúde, valorização dos serviços de atenção primária de saúde como "porta de entrada", para os demais serviços da Rede, atendimento às necessidades de saúde individuais e coletivas da comunidade e universalização da saúde em todas as dimensões do cuidado (promoção da saúde, prevenção de doenças, tratamento, reabilitação) ${ }^{(2)}$.

Ao se abordar a correlação entre a consolidação da RAS e a efetivação dos princípios do SUS, percebe-se que, dentre os objetivos da RAS, são enfatizados a integralidade e a resolubilidade, pois abrangem todas as dimensões do cuidado proposto, devendo, o SUS, oferecer intervenções de promoção, proteção, reabilitação e manutenção da saúde.

Nessa perspectiva, destaca-se que a organização dos serviços em rede visa prestar um cuidado integral, humanizado e contínuo à população. Para tanto, a Atenção Primária em Saúde (APS) ocupa posição estratégica no centro da rede de saúde, devendo estar interligada aos demais pontos de atenção à saúde, de maneira horizontal e interdependente, o que a possibilita coordenar o fluxo e o contrafluxo do atendimento da população $\operatorname{adscrita}^{(2)}$.

Nesse contexto de cuidado, está o enfermeiro contribuindo para a operacionalização da Estratégia de Saúde da Família, sendo reconhecido pela comunidade e pelos profissionais como um profissional confiável, que escuta e busca facilitar e/ou agilizar 0 atendimento, realiza intervenções domiciliares, quando necessário. A inserção desse profissional, nesse contexto, é favorecida por sua disponibilidade, formação generalista, facilidade de comunicação e experiência no planejamento, execução e avaliação das ações de saúde assistenciais, administrativas e educacionais ${ }^{(3-4)}$.

De maneira geral, o papel do enfermeiro na RAS é oferecer atendimento orientado pelos princípios do SUS (igualdade, integralidade e universalidade) aos usuários, visando a promoção e proteção da saúde, prevenção de agravos, tratamento, reabilitação e manutenção da saúde. O enfermeiro desempenha três funções importantes: educacional, assistencial e gerencial. Dentre as funções educativas enfatizam-se: proporcionar educação continuada aos técnicos de Enfermagem e agentes comunitários de saúde, bem como promover educação para a saúde da comunidade. Já as funções assistenciais se concentram na oferta de cuidados individuais e/ou coletivos às pessoas e famílias. Ainda, a gerência de unidades, de programas de saúde, de projetos governamentais e não-governamentais e/ou de ação comunitária, bem como a organização de serviços e planejamento em saúde focados na elaboração, coordenação e execução de projetos de intervenção e impacto no setor de saúde compreendem as funções gerenciais ${ }^{(5)}$.

Assim, no cenário nacional, o enfermeiro é responsável pela continuidade do atendimento integral aos usuários, buscando constituir relações horizontais e oferecer serviços de prevenção, cura e reabilitação para maximizar a saúde e o bem-estar, a partir da centralização das necessidades de saúde da população(6). $\mathrm{Na}$ Inglaterra, que é um dos países que prioriza a universalização da saúde, ampliou-se, desde os anos 90, a atuação do enfermeiro na atenção primária de saúde para se assegurar uma melhor operacionalização da $\operatorname{RAS}^{(7)}$. Ao se considerar a necessidade de se conhecer as ações de enfermeiros atuantes na APS no fortalecimento da RAS numa realidade brasileira, estabeleceu-se o seguinte questionamento: quais ações são desenvolvidas por enfermeiros atuantes na Estratégia de Saúde da Família para o fortalecimento da RAS?

Nessa perspectiva, este estudo objetivou investigar as ações desenvolvidas por enfermeiros para o fortalecimento da RAS numa cidade do Triângulo Sul de Minas Gerais.

\section{MÉTODO}

Trata-se de estudo descritivo, exploratório de abordagem qualitativa, realizado nas unidades de saúde do Distrito 2 de uma cidade do interior 
de Minas Gerais, onde existem três distritos sanitários distintos. A população estimada para o ano de 2017, na referida cidade, é de 328.272 pessoas $^{(8)}$ e a RAS é composta pelas Unidades Básicas de Saúde que atuam como porta de entrada do sistema de saúde, Unidades de Saúde da Família com equipe multiprofissional responsável pelos cuidados básicos e pelas Unidades Matriciais de Saúde, que atendem especialidades básicas $^{(9)}$. Especificamente, no Distrito 2, há um maior número de pessoas em situação de vulnerabilidade social e existe um grande número de equipes de saúde da família (quinze) com 12 enfermeiros atuando, os quais foram suficientes para responder à questão norteadora deste estudo.

Para a obtenção dos dados, utilizou-se de entrevista aberta na unidade de saúde junto aos 12 enfermeiros que se enquadravam nos seguintes critérios de inclusão: atuavam na Estratégia Saúde da Família há, no mínimo, dois anos; estavam lotados nas equipes de saúde da família do Distrito 2 e aceitaram participar da pesquisa por meio da assinatura do Termo de Consentimento Livre e Esclarecido. Utilizou-se, como critério de inclusão, o período de dois anos de atuação para os participantes, pois a intenção era acessar enfermeiros que já estavam adaptados à realidade do serviço desenvolvido na unidade de saúde e na rede municipal de saúde. Foram estabelecidos como critérios de exclusão: enfermeiros que estavam em licença médica ou em afastamento do trabalho por outros motivos, bem como aqueles que não aceitaram participar.

Os profissionais foram contatados por telefone para agendamento de horário, o que ocorreu após autorização da chefia imediata de cada uma das unidades de saúde, sede da respectiva equipe de saúde da família em que atuavam. Nesse contato, também lhes foram apresentados os riscos, benefícios e o objetivo do estudo, assegurando-se também a confidencialidade das informações oferecidas.

Para a elaboração deste instrumento, foram valorizados os resultados de recentes investigações ${ }^{(3-4)}$ que abordaram o trabalho do enfermeiro na APS nos contextos gerenciais, educativos e assistenciais. Com base nessa realidade, o instrumento enfocou o seguinte questionamento: "quais ações gerenciais, assistenciais e educativas são desenvolvidas por você para fortalecer a RAS?"

A coleta de dados ocorreu nos meses de agosto e setembro de 2016 e iniciou-se após a autorização do Comitê de Ética em Pesquisa (CEP), por meio do parecer 1.068.802, do CEP da Universidade de Uberaba, o que foi sucedido pela assinatura do Termo de Consentimento Livre e Esclarecido pelos participantes. Para assegurar a confidencialidade, os extratos das falas dos participantes são apresentados por meio de letra "E" seguida de números crescentes, conforme a ordem das entrevistas.

As entrevistas foram gravadas e transcritas pelas pesquisadoras e, posteriormente, submetidas à Análise Temática que se fundamenta na descoberta dos núcleos de sentidos, que constituem uma comunicação sobre a frequência ou presença de algum significado para o objeto em análise ${ }^{(10)}$. Ao considerar este referencial, realizou-se a leitura sistemática do material organizado previamente e agruparam-se os fragmentos que se repetiam ou possuíam semelhança semântica nos diferentes registros. Posteriormente, categorizaram-se os elementos constitutivos do tema, completando-se as três etapas de análise: pré-análise; exploração do material e tratamento; e interpretação dos resultados obtidos. $\mathrm{Na}$ análise final, foram articulados os dados construídos durante a coleta ao referencial teórico, visando responder ao objetivo da pesquisa ${ }^{(10)}$.

\section{RESULTADOS E DISCUSSÃO}

Dos 12 enfermeiros que participaram deste estudo, todos eram do sexo feminino (100\%), atuantes há mais de três anos (80\%), com idade média de 36,3 anos, casadas $(60 \%)$, não trabalhavam em outro local (80\%), e todas tinham pós-graduação "Iato-sensu" em saúde da família (100\%).

Da sistematização dos dados, evidenciou-se que os participantes consideram que as suas ações cotidianas do trabalho contribuem para o fortalecimento da RAS, mas não acreditam que consolidem a referida Rede, uma vez que o enfermeiro é apenas um componente da equipe de saúde que integra o grupo da Estratégia de Saúde da Família. Assim, dos dados, emergiram as seguintes categorias: comunicação entre os níveis de atenção à saúde; parceria com alguns serviços especializados; promoção da saúde da população e atendimento às gestantes de alto risco.

\section{Comunicação entre os níveis de atenção à saúde}

Tal como ocorre em sistemas de saúde de vários países do mundo, o SUS é um sistema 
fragmentado, cujo acesso é difícil e os cuidados tendem a ter a sua continuidade comprometida, o que dificulta a integralidade da atenção oferecida. De maneira geral, o sistema de saúde brasileiro não corresponde integralmente às necessidades de saúde da população ${ }^{(11)}$.

Ao buscar estruturar a atenção em saúde e minimizar esta fragmentação, foi incorporada, no Brasil, a RAS que busca prestar cuidados contínuos e integrais com resolubilidade, baixo custo, melhor qualidade e de forma humanizada(2).

O sistema de referência e contra referência constitui a estratégia formal de comunicação entre os níveis de atenção à saúde (primário, secundário e terciário) e é considerado uma das intervenções para a consolidação da referida rede. Dessa forma, é imprescindível valorizar a efetividade desse sistema formal de comunicação, que apresenta lacunas no cotidiano da atenção primária, levando à descontinuidade do atendimento, tal como os participantes expressaram:

"A falta de referência e contrarreferência atrapalham o trabalho". (E4)

"Fazemos a referência em impresso para que o usuário não se perca na Rede, mas quase sempre, nunca, recebemos o retorno". (E9)

"Realizamos o preenchimento de guia de referência para encaminhar para outro nível de atenção, porém é muito difícil receber a contrarreferência". (E8)

Nesse contexto, mesmo que o enfermeiro da Estratégia de Saúde da Família tenha utilizado o sistema de comunicação instituído formalmente, há uma interrupção na "cadeia" do cuidado, o que pode ocasionar, nos usuários, a sensação de que suas necessidades não estão sendo atendidas. Essa realidade foi abordada nas seguintes falas:

"Quando atendemos e o paciente entra numa fila que não está andando, encaminhamos para as UPAS e depois este paciente volta para atenção primária e nunca ficamos sabendo o que fizerem com ele, mas ele acredita que a UPA solucionou tudo". (E1)

"Através das demandas que chegam para a equipe, principalmente pelos agentes comunitários de saúde, a equipe discute e referência para tentar solucionar o "problema/caso" e isso faz a população não acreditar na gente". (E10)

A partir dessa realidade, evidencia-se que a interdependência entre os serviços é um princípio não reconhecido pela população e tampouco por muitos profissionais de saúde. As dificuldades de integração das ações da Unidade Básica com os demais níveis de cuidado têm conotação afetiva negativa entre os profissionais, o que se expressa por revoltas/indignação, desânimo/descrédito e impotência frente às limitações na resolubilidade das demandas ${ }^{(12)}$.

Ressalta-se que a associação da interdependência com a articulação entre os serviços é importante para o fortalecimento da rede. Nesse sentido, é importante que os enfermeiros atuantes em todos os níveis de atenção à saúde estabeleçam intervenções propulsoras de um fluxo contínuo no atendimento, como estratégias que viabilizem a melhora na comunicação entre os serviços da $\operatorname{RAS}^{(13)}$.

De maneira geral, a falta de contrarreferência aliada ao descrédito da população possibilitam problemas no cotidiano de trabalho e ainda dificultam a continuidade do atendimento, bem como geram prejuízos na integralidade proposta pela Sistema Único de Saúde ${ }^{(14)}$.

A partir do exposto, evidenciou-se que a interrupção na comunicação entre os níveis de atenção à saúde dificulta o acompanhamento contínuo instituído como intervenção prioritária da atenção primária. Nesse sentido, estudo recente $^{(15)}$ aponta que, quando uma Rede de Atenção à Saúde é fragmentada, a atenção básica não consegue exercer seu papel de centro de comunicação, coordenando o cuidado. Entretanto, os enfermeiros que participaram deste estudo estão estabelecendo parcerias com alguns serviços especializados com vistas à amenização desta realidade, tal como exposto na categoria que se segue.

\section{Parceria com alguns serviços especializados}

Ao lidar com a descontinuidade do atendimento abordada anteriormente e assegurar o atendimento das pessoas assistidas nos respectivos cenários de prática dos participantes desta investigação, foram desenvolvidas parcerias com alguns serviços especializados existentes na rede de saúde do município. Dentre os serviços que melhor estabeleceram as parcerias, destacam-se: Centro de Atenção Integral à Saúde da Mulher (CAISM), Centro de Atenção Psicossocial Infantil (CAPSi), Centro de Atenção Psicossocial Adulto (CAPS) e Unidade de Pronto Atendimento (UPA): 
"Referência para os serviços especializados quando necessário". (E12)

"Parceria com outros departamentos e/ou setores, principalmente CAPS e CAPSi". (E10)

"Encaminhamento da demanda espontânea e agendada para serviços: CAPSi, CAPS, CAISM, dentre outros". (E7)

De maneira geral, essas parcerias colaboram para que os enfermeiros assegurem o acesso das pessoas a outros níveis de atenção à saúde, que oferecem tecnologias adequadas à complexidade das necessidades de saúde identificadas.

Nesse contexto, destaca-se que, no setor saúde, a coordenação dos cuidados pode ser definida como a articulação entre os diversos serviços e ações de forma que, independentemente do local onde sejam prestados, esses serviços sejam sincronizados e voltados ao alcance de um objetivo comum ${ }^{(16)}$, o que corresponde ao princípio de consolidação da rede que estabelece a necessidade de se instituir a atenção primária de saúde como "porta de entrada" para os demais serviços da rede e a universalização da saúde no âmbito de outros níveis de atenção à saúde ${ }^{(2)}$.

$O$ ato de referenciar exige do enfermeiro mais que o simples encaminhamento, pois é necessário que este profissional tenha uma visão holística das pessoas acompanhadas, o que implica o levantamento não apenas de problemas de saúde, mas também de problemas sociais que exigem intervenções articuladas com alguns dos serviços cujas parcerias foram estabelecidas, tal como exposto nas falas que se seguem:

"Acionamento no NASF para casos específicos que necessitem de assistente social". (E7)

"Parcerias com o CRAS em questão de atendimento e demandas sociais". (E3)

"Serviço de agendamento de ambulâncias, nos atendimentos por risco social e não só para atendimentos gerais". (E2)

Ao considerar esta realidade, pode-se afirmar que os enfermeiros da APS que participaram deste estudo utilizam não apenas de parcerias junto aos serviços de saúde para cuidar das pessoas que residem na área de abrangência de sua atuação, mas também valorizam a intersetorialidade para atender a todas as situações que interferem direta ou indiretamente na saúde dessas pessoas, o que indica seu comprometimento com a coordenação do processo de cuidar.
Nesse sentido, em 2011, reforçou-se que cabe à APS, por meio da Estratégia Saúde da Família e do Programa de Agentes Comunitários de Saúde, a coordenação da integralidade do cuidado, articulação das ações de promoção à saúde, prevenção de adoecimento, tratamento e reabilitação a partir dos pressupostos da interdisciplinaridade e intersetorialidade ${ }^{(17)}$.

A atuação intersetorial é condição para que a APS não atue isoladamente e se mantenha como direcionadora dos cuidados da comunidade por ter acesso à realidade biológica, psicológica e social, o que a possibilita agir sobre problemas coletivos e nos determinantes do processo saúdedoença, visando, por conseguinte, a promoção da saúde ${ }^{(18)}$.

É imprescindível que essas parcerias se fortaleçam e se ampliem para assegurar a resolubilidade das necessidades da população, proporcionando a integralidade do cuidado ${ }^{(19)}$. Assim, operacionalizar ações de promoção da saúde que correspondam às necessidades de saúde da população constitui uma das intervenções para a consolidação da rede de saúde e está entre as funções do enfermeiro atuante na atenção primária, valorizada pelos participantes deste estudo.

\section{Promoção da saúde da população}

A promoção da saúde consiste no processo de capacitação individual e coletiva para amenizar os determinantes sociais, objetivando qualidade de vida. Esta proposta é utilizada pelos participantes deste estudo para o fortalecimento da Rede:

"Desenvolvimento de ações de promoção à saúde e prevenção de doenças para que a atenção básica seja satisfatória e evite encaminhamentos desnecessários ou preveníveis para os demais serviços". (E12)

"Ações de promoção, prevenção e reabilitação à saúde com a população". (E7)

"Ações de promoção à saúde com a população local". (E7)

A promoção da saúde parte de uma concepção ampla do processo saúde-doença e de seus determinantes, estando associada a um conjunto de valores que incluem: qualidade de vida, saúde, solidariedade, equidade, democracia, cidadania, desenvolvimento, participação, dentre outros. A articulação de saberes técnicos e populares e a mobilização de recursos institucionais e comunitários para o 
enfrentamento e a resolução dos problemas de saúde são seus elementos essenciais ${ }^{(20)}$.

Dentre as ações de promoção à saúde realizadas pelos enfermeiros, destacam-se os atendimentos domiciliares, a educação em saúde e no ambiente escolar, os atendimentos grupais e individuais, conforme citado nas falas abaixo:

"Atendimento ao usuário, grupos, visitas domiciliares, programa saúde na escola, palestras em sala de espera". (E3)

"Aproveitamento de todos os espaços (atendimento individual, grupos, visita domiciliar)". (E8)

"Parceria entre a atenção básica e a rede escolar, na qual são desenvolvidas ações de promoção em saúde nas escolas". (E6)

"Hiperdia, acolhimento". (E11)

"Reuniões-grupos e ações coletivas com a comunidade, educação continuada". (E4)

A Portaria 648, do Ministério da Saúde, atribui ao enfermeiro da ESF, a realização de uma assistência integral, visando à promoção e proteção da saúde, prevenção de agravos, diagnóstico, tratamento, reabilitação e manutenção da saúde aos indivíduos e famílias do território e, quando indicado ou necessário, no domicílio e/ou nos demais espaços comunitários (escolas, associações), em todas as fases do desenvolvimento humano: infância, adolescência, idade adulta e terceira idade ${ }^{(21)}$.

É perceptível que as ações desenvolvidas pelos enfermeiros deste estudo promovem a saúde e são compatíveis com a legislação do Ministério da Saúde e possibilitam o alcance da resolutividade. Ao considerar essas ações de promoção à saúde, o fortalecimento da Rede se dá a partir de um trabalho articulado, a fim de fortalecer a continuidade do atendimento. Dentre as estratégias da promoção à saúde, os participantes mencionam a informação e educação em saúde em suas falas:

"Informação para a comunidade e integrantes da unidade quanto ao fluxo e funcionamento da rede de atenção para otimização do serviço, e um melhor funcionamento". (E9)

"Orientações sobre funcionamento da rede de atenção, orientação sobre serviços oferecidos em cada nível de atenção, aproveitamento de todos os espaços (atendimento individual, grupos, visita domiciliar) para orientar o que é SUS, direitos e deveres, rede de atenção, funcionamento, o que e quando procurar (serviços/atendimentos)". (E8)
"Informação à população dos serviços disponíveis, respeitar o fluxo da rede de atenção e a necessidade de cada serviço, informando e orientando o paciente para que ocorra um atendimento qualificado e eficiente". (E5)

É notório o fortalecimento da APS, tendo, como um dos principais objetivos, a promoção da saúde como instrumento para a capacitação dos indivíduos para aumentar o controle sobre os determinantes de saúde. Para haver a implementação da promoção à saúde de comunidades e indivíduos, devem-se utilizar ferramentas que visem à capacitação para autonomia, como a educação em saúde ${ }^{(22)}$.

As ações educativas são realizadas com o intuito de construir, junto à população, vínculo e compromisso, buscando beneficiá-la, de forma mais efetiva, com melhorias nas condições de vida e saúde, por meio de um envolvimento fundamentado na corresponsabilização. A prática educativa é capaz de proporcionar aos indivíduos novos hábitos e condutas de saúde, incluindo melhorias no autocuidado(22).

A educação em saúde, portanto, deve estar associada às ações de assistência e culminar na produção de saberes, propiciando, ao indivíduo, autonomia e capacidade de cuidar-se, cuidar de sua família e dos que estão ao seu redor. Com isso, a equipe precisa atentar-se para casos em que a educação em saúde não consegue suprir todas as necessidades, requisitando atenção redobrada, tal como no acompanhamento de gestantes, que corresponde à valorização do atendimento das necessidades de saúde individuais, importante para a consolidação da rede de saúde.

\section{Atendimento de gestantes de alto risco}

Entre as atividades desenvolvidas pelos enfermeiros na APS, há um destaque para o acompanhamento das gestantes de alto risco. Nesse sentido, esse profissional realiza, junto aos agentes comunitários de saúde, desde o cadastramento no Sistema de Informação do PréNatal até o acompanhamento, com ênfase na atenção rigorosa e contínua junto a estas mulheres. Ainda, se necessário, também deve ser feita a busca ativa, tal como expresso nas falas a seguir:

"Acompanhamento e busca ativa de gestantes sem risco e gestantes de alto risco". (E3)

"Acolhimento de gestante de risco". (E11) 
Durante esse acompanhamento, faz-se necessário avaliar as situações que sinalizam riscos gestacionais, que podem ser identificados por meio de patologias, alterações e evoluções desfavoráveis, podendo ser situações que se afastam de um padrão previsível do processo gravídico, vistos como opostos ao normal.

De maneira geral, a categorização da gestação como de "risco habitual" ou de "alto risco" constitui atribuição da equipe de saúde atuante na atenção primária e isso faz com que o enfermeiro articule intervenções para proporcionar acompanhamento especializado para essas mulheres:

"Referência para serviços especializados quando as gestantes necessitam". (E12)

$O$ atendimento de pré-natal de alto risco é concebido para atender as gestantes com o risco gestacional aumentado e serve para manter um serviço especializado para as gestantes que se enquadram na(s) seguinte(s) situação(ões): gravidez gemelar, infecções passíveis de transmissão fetal, história de intercorrência obstétrica prévia (abortamento de repetição, natimorto, perdas fetais), hipertensão, cardiopatias e diabetes ${ }^{(23-24)}$.

Diante desses casos, é necessário que os profissionais da atenção primária mantenham parcerias com os demais níveis de atenção para encaminhamentos e acompanhamentos, com vistas à promoção de um atendimento de maior complexidade:

"As gestantes de alto risco são encaminhadas ao Hospital Universitário e, dependendo do distrito, são encaminhadas ao Hospital Escola da UFTM". (E2)

"Parceria com outros departamentos para encaminhamento de gestante de alto risco". (E10)

Indiscutivelmente, uma das ações dos enfermeiros que participaram deste estudo, para a efetivação do atendimento em rede, é o atendimento às gestantes de alto risco. Nesse sentido, ressalta-se ${ }^{(23)}$ que existem casos que conseguem ser acompanhados e solucionados no nível primário. Entretanto, outros necessitarão de cuidados mais complexos presentes no nível secundário ou terciário, onde existem equipes multidisciplinares constituídas por especialistas de diversas áreas: Enfermeiros, Psicólogos, Assistentes Sociais e Médicos.

Nesse contexto, para que ocorra o fortalecimento da RAS, faz-se necessário a implementação de serviços que atendam às necessidades de saúde individuais e coletivas da comunidade ${ }^{(2)}$. Em recente investigação(25), realizada no Chile, evidenciou-se que o funcionamento da rede de saúde impulsiona a provisão de serviços de saúde.

\section{CONSIDERAÇÕES FINAIS}

De maneira geral, os enfermeiros desenvolvem algumas ações que contribuem para o fortalecimento da RAS, as quais se relacionam aos propósitos da APS e às ações prioritárias para a consolidação da RAS. A promoção da saúde se destacou por meio da educação em saúde como uma ação na qual o enfermeiro busca trabalhar com a comunidade para fortalecer o cuidado de saúde, pois, nela, também são abordadas informações importantes para que as pessoas conheçam o fluxo e a operacionalização da rede de saúde, o que, segundo os participantes, contribui para a operacionalização da rede, uma vez que o desconhecimento das pessoas sobre a referida Rede inviabiliza o seu fortalecimento.

Ao assegurar a resolutividade das demandas sociais e de especialidades, os enfermeiros também estabeleceram parcerias junto a outros serviços de saúde pertencentes a outros níveis de atenção para que as pessoas sejam encaminhadas. Entre as ações desenvolvidas para o fortalecimento da rede, destacou-se $\mathrm{o}$ atendimento de gestantes que exigem diferentes condutas que perpassam pela atenção contínua e rigorosa junto a estas mulheres, pela classificação do risco familiar e por sua referência para outros serviços de saúde com maior densidade tecnológica (nos casos de gestação de risco) e/ou direcionamento para outros setores, quando forem detectadas outras necessidades.

Portanto, os enfermeiros da atenção primária buscam realizar, com excelência, as intervenções específicas da atenção primária, sobretudo a promoção e proteção da saúde, o que possibilita fortalecer este nível de atenção no contexto da Rede. Entretanto, as parcerias estabelecidas se restringem a apenas algumas especialidades (saúde mental, saúde da mulher, urgência e emergência) e ao setor social, o que sinaliza a necessidade de ampliar as parcerias para outros setores (desenvolvimento, educação entre outros) e outros serviços especializados que atendem outras demandas de saúde, tais como: saúde do trabalhador, saúde do idoso, saúde da criança, entre outros. 


\section{REFERÊNCIAS}

1. Ministério da Saúde (BR). Portaria no 4.279, de 30 de dezembro de 2010. Estabelece diretrizes para a organização da Rede de Atenção à Saúde no âmbito do Sistema Único de Saúde (SUS). Brasília, DF: Ministério da Saúde; 2010[citado em 12 maio 2016]. Disponível em: http://www.bvsms.saude.gov.br/bvs/saudelegis/ gm/2010/prt4279 $3012 \quad 2010 . h t m l$

2. Mendes EV. As redes de atenção à saúde. 2nd ed. Brasília, DF: OPAS; 2011[citado em 12 maio 2016]. Disponível em: http://www.paho.org/bra/index.php?option=co $\underline{m}$ docman\&view=download\&category slug=serv icos-saude-095\&alias=1402-as-redes-atencao-asaude-2a-edicao-2\&ltemid $=965$

3. Galavote HS, Zandonade E, Garcia ACP, Freitas PSS, Seidl $\mathrm{H}$, Contarato PC, et al. The nurse's work in primary health care. Esc Anna Nery Rev Enferm. 2016;20(1):90-8. https://doi.org/10.5935/1414-8145.20160013

4. Almeida JHH, Feitosa ANA, Araújo WA, Silva JB, Lourenço LC, Sousa MNA. Primary health care: focusing on the health for the attention of networks. Rev Enferm UFPE on line. 2015;9(11):9811-6.

https://doi.org/10.5205/reuol.8008-72925-1ED.0911201522

5. Oliveira JLC, Papa MAF, Wisniewski D, Inoue KC, Costa MAR, Matsuda LM. Qualidade do cuidado: concepções de graduandos de enfermagem. REME Rev Min Enferm. 2015;19(1):30-42 .

http://www.dx.doi.org/10.5935/14152762.20150003

6. Costa VA, Ramires JCL. A Importância das redes de atenção de saúde para o desenvolvimento da atenção primária em Pirapora. Hygeia (Uberlândia). 2014[citado em 12 maio 2016];10(18):234-49. Disponível em: http://www.seer.ufu.br/index.php/hygeia/article /viewFile/26234/14867

7. Toso BRGO, Filippon J, Giovanella L. Atuação do enfermeiro na atenção primária no Serviço Nacional de Saúde da Inglaterra. Rev Bras Enferm. 2016;69(1):182-91.

https://doi.org/10.1590/0034-7167.2016690124i

8. Instituto Brasileiro de Geografia e Estatística. Cidades. Uberaba . Rio de Janeiro: IBGE; 2010[citado em 19 maio 2016]. Disponível em: https://cidades.ibge.gov.br/brasil/mg/uberaba/p anorama

9. Uberaba. Secretaria Municipal de Saúde. Atendimentos em Saúde. Unidades de Saúde.
Uberaba: Prefeitura Municipal de Uberaba; 2014[citado em 16 maio 2016]. Disponível em: http://www.uberaba.mg.gov.br/portal/acervo/sa ude/arquivos/unidade atencao basica.pdf 10. Minayo MCS. Pesquisa social: teoria, método e criatividade. 29th ed. Petrópolis: Vozes; 2010.

11. Lavras C. Atenção primária à saúde e a organização de redes regionais de atenção à saúde no Brasil. Saúde Soc . 2011;20(4):867-74. https://doi.org/10.1590/S0104-

\section{5}

12. Ministério da Saúde (BR), Conselho Nacional de Secretários de Saúde. A atenção primária e as Redes de Atenção à Saúde. Brasília, DF: Conselho Nacional de Secretários de Saúde, 2015[citado em 12 maio 2016]. Disponível em: http://www.conass.org.br/biblioteca/pdf/AAtencao-Primaria-e-as-Redes-de-Atencao-a-

\section{Saude.pdf}

13. Macedo LMD, Martin STF. Interdependência entre os níveis de atenção do Sistema Único de Saúde (SUS): significado de integralidade apresentado por trabalhadores da Atenção Primária. Interface (Botucatu). 2014;18(51):64759 https://doi.org/10.1590/1807-57622013.0597 14. Moll MF, Goulart MB, Caprio AP, Ventura CAA, Ogoshi AACM. O conhecimento dos enfermeiros acerca as redes de atenção à saúde. Rev Enferm UFPE on line. 2017;11(1):86-93. https://doi.org/10.5205/reuol.9978-88449-61101201711

15. Nora CRD, Junges JR. Política de humanização na atenção básica: revisão sistemática. Rev Saúde Pública . 2013;47(6):1186$200 . \quad$ https://doi.org/10.1590/S00348910.2013047004581

16. Almeida PF, Giovanella L, Nunan BA. Coordenação dos cuidados em saúde pela atenção primária à saúde e suas implicações para a satisfação dos usuários. Saúde Debate . 2012;36(94):375-91.

https://doi.org/10.1590/S0103-

11042012000300010

17. Ferro LF, Emelin CS, Zimmermann $A B$, Castanharo RCT, Oliveira FRL. Interdisciplinaridade e intersetorialidade na Estratégia Saúde da Família e no Núcleo de Apoio à Saúde da Família: potencialidades e desafios. Mundo Saúde . 2014[citado 12 maio 2016];38(2):129-38. Disponível em: https://www.saocamilosp.br/pdf/mundo saude/155562/A01.pdf

18. Pereira KYL, Teixeira SM. Redes e intersetorialidade nas políticas sociais: reflexões 
sobre sua concepção na política de assistência social. Textos Contextos (Porto Alegre). 2013 [citado em 12 maio 2016];12(1):114-27. Disponível em: http://www.redalyc.org/articulo.oa?id=32152737 $\underline{3009}$

19. Costa JP, Jorge MSB, Vasconcelos MGF, Paula $\mathrm{ML}$, Bezerra IC. Resolubilidade do cuidado na atenção primária: articulação multiprofissional e rede de serviços. Saúde Debate. 2014;38(103):733-43.

https://doi.org/10.5935/0103-1104.20140067

20. Azevedo E, Pelicioni MCF, Westphal MF. Práticas intersetoriais nas políticas públicas de promoção de saúde. Physis (Rio J.). 2012;22(4):1333-56.

https://doi.org/10.1590/s0103-

\section{5}

21. Ministério da Saúde (BR). Portaria № 648, de 28 de março de 2006. Aprova a política Nacional de Atenção Básica, estabelecendo a revisão de diretrizes e normas para a organização da Atenção Básica para o Programa Saúde da Família (PSF) e o Programa Agentes Comunitários de Saúde (PACS). Brasília, DF: Ministério da Saúde; 2006[citado em 12 maio 2016]. Disponível em: http://bvsms.saude.gov.br/bvs/saudelegis/gm/20 06/prt0648 28032006 comp.html

22. Mallmann DG, Galindo Neto NM, Sousa JC, Vasconcelos EMR. Educação em saúde como principal alternativa para promover a saúde do idoso. Ciênc Saúde Coletiva. 2015;20(6):1763-72. https://doi.org/10.1590/1413-

\subsection{4}

23. Demitto MO, Gravena AAF, Agnolo CMD, Antunes MB, Pelloso SM. Gestação de alto risco e fatores associados ao óbito neonatal. Rev Esc Enferm USP. 2017;51:e03208. https://doi.org/10.1590/s1980-

\section{0x2016127103208}

24. Lenz MLM, Flores R, organizadores. Atenção à saúde da gestante em APS . Porto Alegre: Hospital Nossa Senhora da Conceição; 2011[citado em 12 maio 2016]. Disponível em: http://www2.ghc.com.br/GepNet/publicacoes/at encaosaudedagestante.pdf

25. Ferrada AO, Méndez CA. Implementation de las redes asistenciales de salud en Chile: percepciones de los profesionales de la salud. Rev Gerenc Polit Salud . 2013 [citado 20 dez 2017];12(24):100-13. Disponível em: http://www.scielo.org.co/pdf/rgps/v12n24/v12n 24a07.pdf
Nota: Agência de desenvolvimento: PIBIC-UNIUBE.

Recebido em: 06/08/2017

Aprovado em: 24/01/2018

Endereço de correspondência:

Marciana Fernandes Moll

Av. Nenê Sabino, 1801- Bairro Universitário

CEP: 38055-500 - Uberaba/MG - Brasil

E- mail: mrcna13@yahoo.com.br 